\title{
On Mass Loading and Dissipation Measured with Acoustic Wave Sensors: A Review
}

\author{
Marina V. Voinova \\ Department of Physics, University of Gothenburg, 41296 Göteborg, Sweden \\ Correspondence should be addressed to Marina V. Voinova, marina.voinova@physics.gu.se
}

Received 31 December 2008; Accepted 18 April 2009

Recommended by Michele Penza

\begin{abstract}
We summarize current trends in the analysis of physical properties (surface mass density, viscosity, elasticity, friction, and charge) of various thin films measured with a solid-state sensor oscillating in a gaseous or liquid environment. We cover three different types of mechanically oscillating sensors: the quartz crystal microbalance with dissipation (QCM-D) monitoring, surface acoustic wave (SAW), resonators and magnetoelastic sensors (MESs). The fourth class of novel acoustic wave (AW) mass sensors, namely thin-film bulk acoustic resonators (TFBARs) on vibrating membranes is discussed in brief. The paper contains a survey of theoretical results and practical applications of the sensors and includes a comprehensive bibliography.
\end{abstract}

Copyright (C) 2009 Marina V. Voinova. This is an open access article distributed under the Creative Commons Attribution License, which permits unrestricted use, distribution, and reproduction in any medium, provided the original work is properly cited.

\section{Introduction}

This paper introduces readers to microelectronic sensor devices based on surface acoustic waves (SAWs-sensors, Section 2) and on bulk acoustic waves (BAWs-sensors, QCM, Section 4, and thin-film bulk acoustic resonators (TFBARs, Section 5), addressing their physical principles, theory, and selected examples of practical applications. In the third section, we consider the magnetic analogue of SAW resonators, the so-called magnetoelastic sensors (MESs), ribbon-like oscillators that can be successfully used for remote query sensing of (bio)chemical or environmental changes. The fifth section with a brief discussion of a novel TFBAR technique concludes the first part of the paper. In the second part we provide selected examples of chemical and biological applications of the AW-based sensors and conclude with the outlook.

The mechanically oscillating sensors (or acoustic wave (AW) resonators) are commonly used for monitoring a negligible amount of surface mass. Also, AW devices enable researchers to measure other physical parameters such as thickness, viscosity, elasticity, and effects of roughness/sliding friction on thin films of different nature.

The main progress has been made in studying adsorbed molecular films, thin polymer films, and bioorganic layers on surfaces. Successful characterization of biomolecular systems in their natural aqueous environment is the important objective of biosensoring. The quartz crystal microbalance (QCM) and surface acoustic wave (SAW) sensors, which can operate in fluids, straightforwardly offer such a possibility. A polymer coated or biochemically modified sensor surface forms a "tolerant" biological interface which is available for further mounting of biomolecular complexes exposed to water solution. Biosensor systems based on functionalized QCM and SAW devices have become a suitable tool for in situ measurements in biofluids, in particular, for on-line detection of immunological reactions. The vast literature on SAW and QCM experiments and theory reveals a strong and continuous interest in this topic.

A special type of quartz resonator, the so-called electrochemical QCM (EQCM), is a sensor enabling detection of chemical parameters via electrical conductivity measurements both in the gaseous phase, vapours, and in various liquids. Typically EQCM is based on usage of electroconducting polymer arrays as a smart coating of the sensor surface.

Particular attention is focused now on biosensors and bioelectronics, biomedical and environmental applications of the AW sensors. A brief overview of the biology-related achievements is given in Section 6 (Chemical and Life Science Applications). 
New developments are coming now due to the rise of governmental and public interest to the terrestrial ecology and extraterrestrial research. A great benefit of the quartz oscillators is that their operation is gravity independent so they can be successfully used in space exploration research [1-3]. "The most widely used complex measuring instrument in science is still the balance." (NASA, http://trs-new.jpl.nasa.gov/dspace/handle/2014/39937.) QCM-based sensors are planned to be used [4] during The European ExoMars Rover Mission, which is prepared by ESA for a launch in 2009 and is devoted to the "search for life or traces of it, and of water, as a precondition for the existence of highly complex organisms." (ESA MISSION, http://www.esa .int/SPECIALS/ExoMars/SEM10VLPQ5F_0.html.)

Another challenge is to use the quartz sensors for ecosystem observations and pollution control [5]. Terrestrial and marine applications of the QCM include coastal developments [6], aquatic microbial probes [7], studies of the ocean bio-foul [8], and safety and security [9].

For the convenience of reading, we organized the paper in the following way. Each chapter contains brief information about the basic principles, theory, and applications of the AW devices of a particular type. A special chapter on chemical and life science applications contains illustrations and basic principles of surface nanocomposite functionalization of AW sensors. The references are placed at the end of the paper. The list of companies that manufacture acoustic sensors of each type is appended after the list of references (see Appendix $6.4)$.

1.1. Physical Principles of Acoustic Wave (AW) Sensors. A biosensor device can be defined as an analytical microelectronic device either based on a combination of biomolecular sensor elements and a solid state transducer or applied to living organisms to monitor biological and biochemical processes in there [10]. Biocomponents conjugated with a solid state transducer provide a high selectivity to chemical analytes to such a hybrid device while the transducer element (e.g., metal electrode) can measure with a high precision and then convert the input chemical information into a digital electronic signal. In biosensors, common transducers are amperometric, potentiometric, and conductiometric devices, optical sensors, thermal detectors, and acoustic sensors.

An acoustic biosensor is a type of a sensor device, where the transducer element is based on a solid slab of piezoelectric material that can generate acoustic waves in the sensor's substrate. The most popular material for the acoustic sensor substrate is a quartz crystal cut in specific crystallographic directions (the so-called AT-cut and ST-cut quartz plates) so that the angle of the plate in the quartz crystal supports a shear deformation. We consider two different types of piezoelectric resonators, namely, bulk acoustic waves (BAWs) sensors based on a thickness shear vibration mode for AT-cut quartz and surface acoustic waves (SAWs) devices with a shear horizontal (SH) mode for ST-cut quartz and Rayleigh surface wave (RSAW) sensors.

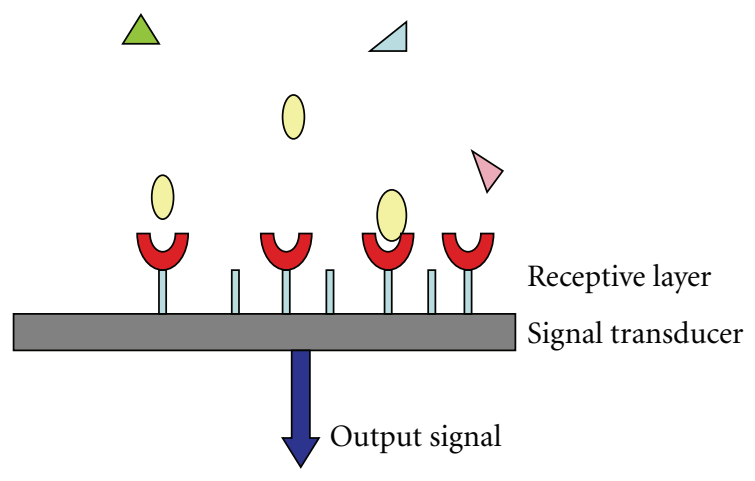

FIGURE 1: Principle of biosensor construction. Typically a biosensor device consists of a sensitive biomolecular layer (grafted polymers, supported membranes, bioreceptors, nucleic acids, enzymes or antibodies) immobilized onto the surface of solid substrate (transducer) that can measure the output signal (e.g., electrical catalytic current in enzyme-based biosensors, mass changes in acoustical piezoelectric resonators, etc.).

These devices are widely used now not only in a gas but also in liquid environments, in particular for biomolecular detection in biological fluids and immunosensor applications. In parallel with quartz resonators, we discuss two novel types of AW devices, namely, silicon-based resonators, (TFBARs) and magnetoelastic ribbon-based sensors (MESs) that recently received considerable attention for acoustic sensor technology.

The sensitivity of the acoustic sensor is proportional to the energy in the propagation path. In the bulk acoustic wave mode of propagation, the energy disperses from the oscillating surface of the transducer though the coating material. The ratio between the energy dissipated during one period of oscillation and the energy stored in the oscillating system determines the quality factor ( $Q$-factor) of the resonator, an important characteristic of the device. In surface acoustic wave sensors, the energy is trapped near the oscillator surface, which increases the sensitivity of the device in measurements of the surface mass of thin films (see Section 2, in particular, Section 2.2).

\section{Surface Acoustic Waves Sensors}

2.1. Surface Acoustic Wave (SAW) Resonators: SH-SSW and RSAW. Primarily, surface acoustic wave (SAW) sensors have been designed and used for the detection of (toxic) chemical components in the gaseous phase. In the latest developments many research groups have used SAW-based devices for biological sensing in liquid environments as well.

The last two decades of intensive experimental efforts in the field of SAW-based biosensors include the design and fabrication of horizontally polarized shear waves- (SH SAW-) based devices, Love wave sensors, and Rayleigh-type surface acoustic wave sensors (RSAW), mass-sensitive devices operating in the high frequency range between $100 \mathrm{MHz}$ and a few GHz. The usage of interdigital transducers (IDT) for the generation and detection of surface acoustic waves allows a 
strong confinement of the acoustic energy to the surface of the piezoelectric slab regardless of its thickness [11]. Due to this confinement, the shift in acoustic wave velocity $\Delta v$ due to the surface mass change is very sensitive to the variations in viscosity, elasticity and electrical conductivity of the deposited layer. SAW-based sensors are widely used for detecting gases and volatile compounds. In liquids, after coating with layers of surface bound receptive layers (such as adsorbed antibodies or covalently bound hydrogel bound layers), these sensors can be used as biosensors by themselves or can be integrated with microfluidic systems $[1,12]$. The interested readers can find further information on the latest technical developments in SAW biosensors in the excellent review by Länge et al. [13] and in the valuable product review article by Harris [9].

2.2. Love Wave Sensors. Surface waves on plane interfaces have amplitude which decays exponentially with the normal distance from the solid surface on which they propagate [14]. The so-called Love waves are of special interest for the SAW design. The related physical effect was originally discovered by Love. (A.E.H. Love. Some Problems of Geodynamics Cambridge University Press, Cambridge (1911).) He observed an effect caused by earthquake waves far from the epicenter due to the lower acoustic wave velocity of waves propagating along the stratified geological layers. Love wave device, a type of SH SAW sensors, is based on the finding that losses of acoustic energy to the bulk substrate can be drastically reduced by a resonating surface bound layer. Simultaneously, an acoustic bound layer resonance leads to a higher sensitivity of the sensor to surface mass. A Love-wave resonance is excited when the layer has optimal thickness and appropriate material parameters [11, 13]. polymethyl methacrylate (PMMA) coating was found a suitable wave-guiding material for these purposes. The first Love wave biosensor on PMMA-on-quartz-crystal was reported by Gizeli et al. [15]. PMMA served as a host matrix enabling a further mounting, for example, a coating with additional gold film, followed by thiol SAM- or a peptide layer deposition.

Two different types of biosensors based on Love wave devices are presented in $[12,16-18]$; see also papers $[11,13]$ for a review.

2.3. SH SAW Sensors. The SH SAW sensors can be constructed on coated piezoelectric substrates like quartz, lithium niobate and lithium tantalate cut in a special way (e.g., Figure 2, shows the Y-cut X propagating lithium tantalate substrate. Two acoustic modes are excited by the electrodes in this crystalline direction). The phase velocity of surface shear waves, $V$, is lower than that of bulk shear waves, $V_{0}$ in the semi-infinite elastic half-space (i.e., the substrate). This difference in phase velocity can be monitored with high accuracy in SAW devices. Sensitive coating with polymer films results in the observation of resonant conditions in both modes as the layer thickness is increased. The shift in phase velocity $\Delta V=V-V_{0}$ and the damping coefficient $\Gamma$ ,simply the imaginary part of the SAW wave vector $q$, depend

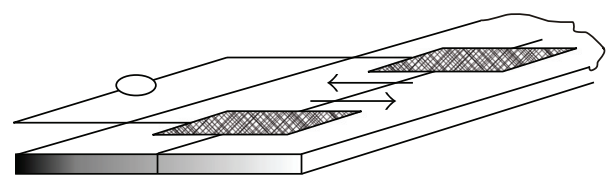

FIgURE 2: Sketch of the geometry of an SH SSW-based sensor.

on the surface mass and mechanical parameters of the layer coating the oscillator's surface.

From the analysis of the dispersion equation at no-slip boundary conditions between the coating thin layer and the resonator surface, one can find for the phase velocity shift and for the damping coefficient of the resonator the following expressions:

$$
\begin{aligned}
\frac{\Delta V}{V} & \approx 2\left(\frac{\omega \rho_{s}}{V_{0} \rho_{0}}\right)^{2}, \\
V_{0} & =\sqrt{C_{44} / \rho_{0}}=\text { const }, \\
\Gamma & =\frac{q_{0} \omega^{3} \rho \eta}{\mu_{0}} .
\end{aligned}
$$

Here $\rho_{0}$ is the density of the crystal substrate, $\mu_{0}$ is the elastic shear modulus of the substrate, $\omega$ is the oscillator frequency, $\rho_{s} \equiv \rho h$ is the surface mass of the thin coating film, and $\rho, \eta$, and $h$ denote the film density, shear viscosity and thickness, respectively. In fact, the no-slip condition, for example, when the LB film is covalently bound to the substrate, corresponds to the Love wave polarization.

The coating film in biological applications can be a protein adsorbed layer, Langmuir-Blodgett film or supported lipid membrane. In the case of a double layer coating with different surface densities of the sublayers, (1) can be generalized to [19]

$$
\frac{\Delta V}{V} \approx \frac{1}{2}\left(\frac{\omega \rho_{s}}{V_{0} \rho_{0}}\right)^{2}\left(1+\frac{\rho_{s 1}}{\rho_{s 2}}\right)^{2}
$$

Here $\rho_{s 1}, \rho_{s 2}$ values represent the surface density of the sublayers in the film. The result for the damping coefficient for the thin bilayer coating is given by the formula [19]

$$
\Gamma=\Gamma_{\text {monolayer }}\left\{1+\frac{\eta_{2 s}}{\eta_{1 s}}\left(1+\frac{\eta_{1 s} \rho_{1 s}}{\eta_{2 s} \rho_{2 s}}\left(1+\frac{\eta_{2 s}}{\eta_{1 s}}\right)\right)\right\} .
$$

The surface mass and viscosity effects in biomolecular films can be measured also in other types of acoustic experiments, using the so-called bulk acoustic waves, or the BAW mode of propagation.

2.4. Rayleigh Surface Acoustic Wave (RSAW) Sensors. RSAWbased devices as it follows from their name are based on Rayleigh wave polarization. The analysis of the dispersion equation for the Rayleigh wave polarization in the limit of strong coupling between a surface bound layer and a solid substrate and when only mass loading is taken into account gives the following formula for the change $\Delta V$ of 
the Rayleigh wave velocity $V_{R}$ due to the presence of surface bound layer $\rho_{S}$ :

$$
\frac{\Delta V}{V_{R}}=k \frac{\rho_{S}}{\rho}\left(\frac{V_{R}}{c_{t}}\right)^{2}
$$

This result has been corrected for soft layers by accounting for the elastic moduli of the layer material (this correction for the Rayleigh polarization has been reported elsewhere [20]).

The first experimental realization of RSAW-based biosensors has been reported by Wohltjen and Dessy in 1979 [21]. The interested readers can find an account of later RSAW developments in gas sensing [22-24] and biosensors in [25] and in the extensive review by Länge et al. [13] (see also the outlook section, for microfludic applications [26-29]).

\section{Magnetoelastic Sensors (MESs)}

Magnetoelastic ribbon-like oscillators are considered as the magnetic analogue of surface acoustic wave sensors [30-44]. Typically, they are made of amorphous

iron- or nickel-based "ribbons" (or thick films) such as Metglas (means metallic glass) (Allied Signal Corporation, Honeywell, http://www51.honeywell.com/honeywell/), a novel type of magnetostrictive materials (Figure 3). The big advantage of ferromagnetic magnetostrictive ribbons is the small size $(5.0 \mathrm{~cm} \times 1.0 \mathrm{~cm} \times 30 \mathrm{mkm})$ of the strips and the low materials cost making this microsensor technology suitable for in situ measurements of chemical agents and contaminants in various media. In such magnetoelastic sensors (MESs) the longitudinal elastic standing waves are excited by an ac magnetic field at the mechanical resonance frequency when the conversion of the magnetic energy into elastic energy is at maximum [30].

Magnetoelastic thick film sensors also have the important advantage of enabling wireless operation and monitoring and are important new tools in sensoring and biosensoring [31-44].

The fundamental resonance frequency of a ribbon-like MES is given by

$$
f_{0}=\frac{1}{L} \sqrt{\frac{E}{\rho}}
$$

Here $L$ is the ribbon length, $E$ is the Young's modulus, and $\rho$ is the ribbon material density.

For a small mass gain attached to the one side of the oscillator, the resonance frequency change is equal to

$$
\Delta f=f-f_{0}=-f_{0} \frac{\Delta m_{S}}{2 M} .
$$

Taking into account the elastic stress in the case of a uniform coating film, the ratio of the measured frequencies before and after the coating application is given by the formula [31]

$$
\frac{f}{f_{0}}=\sqrt{\frac{M}{m_{\mathrm{tot}}}+\left(1-\frac{M}{m_{\mathrm{tot}}}\right) \frac{E_{f} / \rho_{f}}{E / \rho}},
$$

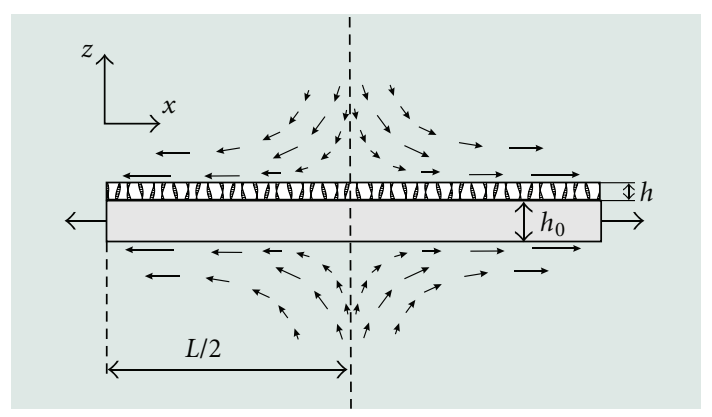

Figure 3: Sketch of a magnetoelastic sensor. A schematic (crosssectional) illustration of longitudinal vibrations of a ribbon-like elastic plate coated with an organic layer and immersed into a bulk viscous liquid. The geometry of the system is shown directly on the figure body (picture is taken from [45]).

where the index " $f$ " corresponds to the film parameters, $M$ is the mass of the sensor, and $m_{\text {tot }}$ is the total mass after coating.

Formula (7) enables us to deduce the film elasticity $E_{f}$ from experimental data in gas or vacuum. In bulk liquid measurements, the change in resonance frequency of the magnetoelastic ribbon of density $\rho$ and thickness $d$ is proportional to the square root of the product of the density $\rho_{l}$ and the shear viscosity $\eta$ of the liquid $[31,32]$ :

$$
\Delta f=-\frac{\sqrt{\pi f_{0}}}{2 \pi \rho d} \sqrt{\eta \rho_{l}}
$$

The quality factor $Q$ for low viscosity liquids is related to the damping and has the same form as the expression for the Q-factor for QCM resonators, namely

$$
\frac{1}{Q}=\frac{1}{Q_{0}}+\frac{1}{\rho d \sqrt{\pi f_{0}}} \sqrt{\eta \rho_{l}} .
$$

The expressions (8) and (9) give a correct result only in the case of an ideally smooth oscillator surface. The correction for the roughness which increases the viscous damping contribution has been published elsewhere [32]. Another correction factor is attributed to the effects of geometry that has been recently found in [45].

Among chemical and biomedical applications of wireless MES one can mention the remote query environmental monitoring [30,33,34, 42], bloodstream chemistry [38, 44], safety [39], bioaffinity detection [40], and many others.

Future developments in this field include in situ and in vivo experiments for example, gastric $\mathrm{pH}$ monitoring through the digestive tract [36] or measurements of enzymatic reactions in tissues and organs [37].

\section{Bulk Acoustic Waves Resonators}

4.1. The Quartz Crystal Microbalance with Dissipation (QCMD) Monitoring. In addition to "genuine" surface waves, another type of shear waves can propagate in the plane of an overlayer-substrate interface. These are bulk acoustic waves (BAWs). 


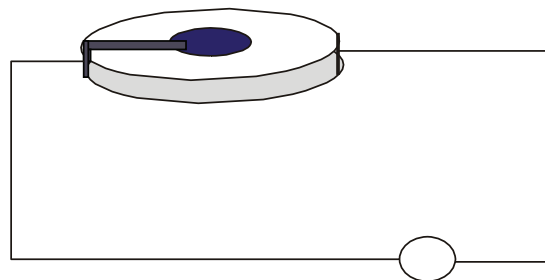

(a)

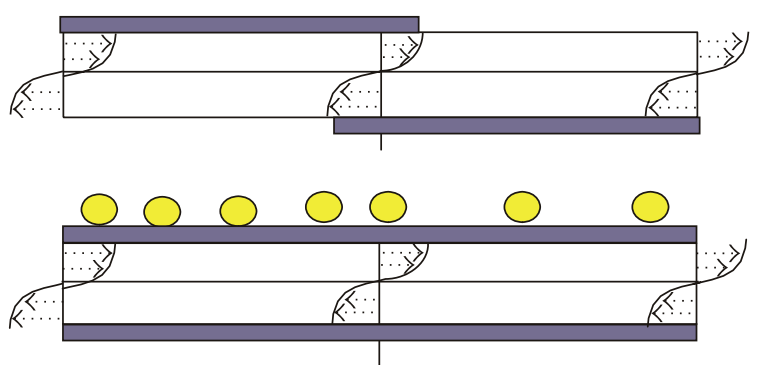

(b)

FIGURE 4: Sketch of a quartz crystal resonator: (a) schematic depiction of a quartz disk with deposited electrodes; (b) side view of the resonator; shear deformation produced by the electric field. This type of acoustic sensor operates in a BAW mode, when acoustic shear waves propagate in the direction normal to the quartz plane.

The acoustic BAW-based sensor shown in Figure 4 consists of a thin disk of AT-cut quartz crystal plate with metal (typically, gold) electrodes deposited on either side. When driven electrically to resonate, the crystal produces shear mechanical stress, acoustic shear waves which propagate out from the crystal faces. The fundamental frequency of the device in vacuum is given by formula

$$
f_{0}=\frac{1}{2 h_{0}} \sqrt{\frac{\mu_{0}}{\rho_{0}}}
$$

where $h_{0}$ is the thickness of the quartz plate, $\rho_{0}$ is its density, and $\mu_{0}$ is the shear elastic modulus of quartz (e.g., the fundamental resonance of a quartz oscillator is $5 \mathrm{MHz}$ for a $0.33 \mathrm{~mm}$ crystal).

It has been found that the resonant frequency of such quartz plates decreases when its surface is coated with an overlayer constituting a mass load. The experimental fact that the changes of the surface mass are proportional to the shift of the crystal resonant frequency is the fundamental operational principle of quartz crystal microbalance (QCM).

Quartz crystal microbalance (QCM) is a thickness shear mode resonator which fundamental frequency is sensitive for even negligible changes in its surface mass $\Delta M$ (a microbalance principle of operation). For a small amount of mass deposited onto the QCM surface, the shift of resonance frequency is proportional to the surface mass $\Delta M$ of the deposit (Sauerbrey, 1959) [46]:

$$
\frac{\Delta f}{f_{0}} \approx-\frac{\Delta M}{m_{0}} .
$$

Equation (11), known as Sauerbrey's formula for the decrease of the resonance frequency, can only be used for thin films rigidly attached to the QCM surface.

When one face of the device is brought into contact with an aqueous solution, the shear waves are strongly absorbed within a few microns of the surface. The crystal detects changes in viscosity and density in this region by monitoring the change in its resonance frequency and damping due to the viscous dissipation in the overlayer. Experimentally, it is convenient to obtain this viscous dissipation as the width of the resonant frequency or as the dissipation factor $D[47-52]$ :

$$
D=\frac{E_{\text {dissipated }}}{2 \pi E_{\text {stored }}}=\frac{1}{\pi f \tau} .
$$

Here, $\tau$ is the time constant for the decay of the vibration amplitude, the dissipation factor value is equal to the reciprocal quality factor $Q$.

When the rigid film is replaced by a Newtonian bulk liquid of shear viscosity $\eta$, the QCM resonant frequency shift is given by the following expression (Kanazawa and Gordon, 1985, [51]):

$$
\Delta f \approx-\frac{1}{2 \pi m_{0}} \sqrt{\frac{\rho \eta \omega}{2}},
$$

so the total response $\Delta f$ for the system of a QCM rigid filmimmersed-in-water is the sum of contributions from the film surface mass (11) and from the viscous contribution of water (13). However, a theoretical analysis shows that for nonrigid deposits this result is not valid. It was found that the viscous loss of energy in the overlayers causes the deviation from ideal behaviour and results in a nontrivial reduction in measured surface mass of the film, $M_{s}=M(1-\alpha)$, where $M$ is the "true" film mass and the viscous correction factor $\alpha$ depends on the mechanical properties of both the overlayer material and of the aqueous solution $\eta, \rho$. This reduction in resonance frequency has been predicted for the first time for the special case of viscous membrane bilayers [19]. Meanwhile, this result has been generalized for the case of arbitrary soft (viscoelastic) deposits [53]. For soft deposits, it was found that the reduction in liquid phase QCM measurements of surface mass $M_{s}$ comparing with the "true" mass Mof the film is:

$$
M_{s}=M\left\{1-\frac{\eta_{2} \rho_{2} \omega}{\rho_{1}} \frac{G^{\prime \prime}}{G^{2}+G^{\prime \prime 2}}\right\}, \quad M=\rho_{1} h_{1} .
$$

Here $G^{\prime}$ is the storage modulus and $G^{\prime \prime}$ is the loss modulus of the soft (viscoelastic) overlayer, index "1" denotes the film properties, and index " 2 " corresponds to the liquid phase, respectively. 
4.2. Viscoelasticity of Polymeric Overlayers: Voight (Viscoelastic Solid) Model. For the QCM-D characterization of macromolecular multilayer assemblies such as polymer "brush" films, adsorbed protein layers, or tethered supported membranes the mechanical properties of a viscoelastic material must be taken into account (for review, see, e.g., Johannsmann's paper [49]). A big family of polymeric coatings which conserve their shape and do not flow is well described by homogenous layers of Voight viscoelastic solids (a model element with parallel arrangement of a spring (for elasticity) and a dashpot (for viscous damping)). Even this relatively simple theoretical model has allowed us to demonstrate the most important consequences of adsorbate viscoelasticity [54] in terms of a deviation from the Sauerbrey relation relevant for a gas environment and ultrathin layers.

For the QCM operating in a gas phase or vacuum, it has been found that for the no-slip boundary conditions and in special case of thin soft overlayer:

$$
\begin{aligned}
\Delta f & \approx-\frac{1}{2 \pi \rho_{0} h_{0}} h p \omega\left(1+\frac{2 h^{2}}{3 \delta^{2}\left(1+\chi^{2}\right)}\right), \\
\Delta D & \approx \frac{2 h^{3} \rho \omega}{3 \pi f \rho_{0} h_{0}} \frac{1}{\delta^{2}\left(1+\chi^{2}\right)}, \\
\chi & =\mu / \eta \omega, \\
\delta & =\sqrt{\frac{2 \eta}{\rho \omega}} .
\end{aligned}
$$

In the case of a viscoelastic bilayer under a bulk layer of Newtonian liquid (index " $B$ "), the calculated QCM response is given by the formulae:

$$
\begin{aligned}
\Delta f \approx-\frac{1}{2 \pi f \rho_{0} h_{0}}\left\{\frac{\eta_{B}}{\delta_{B}}\right. & \\
& \left.+\sum_{i=1,2}\left[h_{i} \rho_{i} \omega-2 h_{i}\left(\frac{\eta_{B}}{\delta_{B}}\right)^{2} \frac{\eta_{i} \omega^{2}}{\mu_{i}^{2}+\omega^{2} \eta_{i}^{2}}\right]\right\},
\end{aligned}
$$

$$
\Delta D \approx \frac{1}{\pi f \rho_{0} h_{0}}\left\{\frac{\eta_{B}}{\delta_{B}}+\sum_{i=1,2}\left[2 h_{i}\left(\frac{\eta_{B}}{\delta_{B}}\right)^{2} \frac{\mu_{i} \omega}{\mu_{i}^{2}+\omega^{2} \eta_{i}^{2}}\right]\right\} .
$$

Formulae (15)-(18) the primary result of our early work [54]. The results of the theory can be applied for an analysis of QCM-D acoustic measurements of surface densities and/or shear viscosity and elasticity for thin polymeric coatings, adsorbed layer of proteins, or supported membranes after the transferring onto the solid substrate and in both the liquid and gaseous experimental conditions (Q-Sense). Also, the results are useful for understanding the shear response of biological bilayer membranes with a different composition of upper and lower monolayers corresponding to the observed compositional asymmetry of red blood cell membranes supported by an elastic substrate (protein network), bioaffinity measurements, immunological reactions, diatoms adhesion, and many others (see for review, [55-61]).

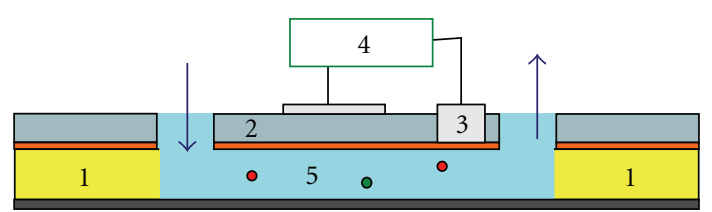

FIGURE 5: Sketch of the TFBAR structure in contact with liquid: 1-Si wafers, 2-AIN piezoelectric layer (aluminium nitride) piezoelectric layer, 3-Al electrodes, 4-analyzer, 5-liquid in a microfluidic system in contact with the resonator (for review, see [68]).

4.3. QCM as a Mechanical Spectroscopy Tool. The quartz crystal microbalance can naturally be considered as a mechanical spectroscopy tool which can monitor the viscoelastic material properties by probing samples performing small-amplitude oscillations at prescribed frequency $\omega$ [62]. Several groups have reported on the application of the QCM to the viscoelastic characterization of thin organic overlayers in a frequency range from a few $\mathrm{Hz}$ to about $100 \mathrm{MHz}$ (for a review, see [49] and references. therein). In particular, as a mechanical spectroscopy tool, the quartz crystal resonator can probe the storage and loss moduli of a thin soft polymeric sample such as adsorbed biomolecular layers, polymer cushions or liquid crystalline polymers in small amplitude oscillations where polymer materials exhibit linear viscoelasticity [62]. The experiments with thin films are of particular interest from the point of view of surface science since the viscoelastic moduli of a thin layer sample can sufficiently differ from the corresponding bulk magnitude.

\section{Thin-Film Bulk Acoustic Resonators}

Thin-film bulk acoustic resonators (TFBARs) are based on a vibrating small solid membrane that is fabricated onto a silicon substrate that can be functionalized with a sensing nanocomposite coating [63, 64] (Figure 5). TFBARs have attracted experimental attention since this novel technique is a high-Q-factor wireless system operating in the microwave region from 1 to $10 \mathrm{GHz}$ [65-69]. This type of resonators meets the requirement for low-cost and small size sensors with the benefit of a high working frequency in comparison with similar SAW-based devices. The main progress in TFBARs has been triggered by FBAR filters applications for the telecom industry, particularly, in mobile phones [66-68].

Silicon-based TFBARs can be considered as a highfrequency version of the quartz crystal microbalance [64].

In gas measurements, the mass sensitivity $S_{m}$ of the TFBAR oscillator increases with the square of the operating frequency:

$$
S_{m}=-\frac{2 f_{0}^{2}}{\rho_{0} h_{0}^{2}}
$$

and the surface mass density $\Delta m_{S}$ is proportional to the measured sensor signal $\Delta f$ :

$$
\Delta f=-S_{m} \Delta_{m s}
$$


Here $f_{0}$ is the resonant frequency of the TFBAR, $\rho_{0}, h_{0}$ are its density and thickness, respectively.

Depending on the structure, surface geometry, and composition of the sensing coatings, the mass sensitivity of the TFBAR systems can be sufficiently improved for nanocomposite layers embedded into a host polymer or another organic coating, for example, cadmium arachidate matrix with embedded SWCNTs arrays [64].

In vapour and liquid TFBARs measurements, the effects of viscosity must be taken into account [66]. Recently, fabrication and successful usage of TFBARs for biosensor applications have been reported in [68]. For the analysis of the experimental data of TFBARs in liquids, the theoretical models of Stockbridge [47] and Kanazawa and Gordon [51] have been used [66].

For correct interpretation of TFBARs measurements of viscoelastic and biological materials in liquid applications, formulae (14)-(18) can be of great help.

There are certain limitations of liquid and biological applications of TFBARs. Due to the higher operation frequency, TFBARs resonators are much thinner than QCMs and thus, more fragile. In general, the important engineering challenge is the optimization of the resonators' geometry from the view point of the sensitivity-to-noise ratio [66].

This TFBARs technique is a very promising method in view of biosensor purposes and, in particular, a combination of TFBAR devices in microfluidic arrays opens a new route towards lab-on-chip applications, microanalysis, and small size immunosensors.

\section{Selected Examples of Applications of AW Sensors}

6.1. Chemical and Life Science Applications of SAW-Based and BAW-Based Sensors. Biosensors based on acoustic resonators, like other bioanalytical methods such as immunoassays or enzyme assays, use biological compounds as the receptive overlayer that can distinguish between adsorbed analytes. Recent applications of thin layered films like selfassembled monolayers and supported bilayers (Figure 6(a)), Langmuir-Blodgett (LB) films (Figure 6(b)), protein monolayers (Figure 7) and multilayers of complex biomolecular architecture (Figure 8 ) as receptive layers in biosensors have stimulated detailed studies of the physical properties of such structures. Among novel promising nanomaterials for the surface functionalization of sensors one can list also stimuliresponsive polymer coatings, synthetic receptors, and carbon nanotubes. In particular, a thin-film bulk acoustic resonator functionalized with a nanocomposite LB layer of carbon nanotubes has been recently suggested as a fast response sensor for high-performance gas detection $[63,65]$.

With these model systems it is possible to construct multilayer amphiphilic films with controlled monolayer thickness and to create different coupling strengths between the adsorbed film and the chemically modified substrate. Specifically measurements of surface density and viscosity of these films are important both scientifically and, for example, with respect to their possible application in acoustical

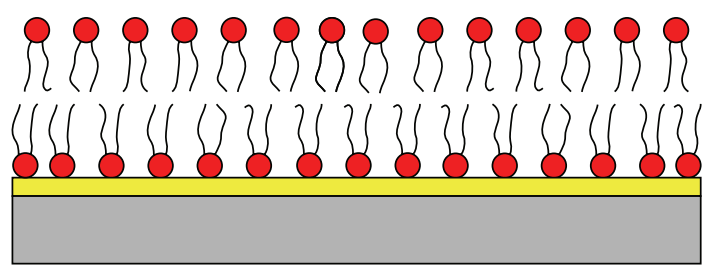

(a)

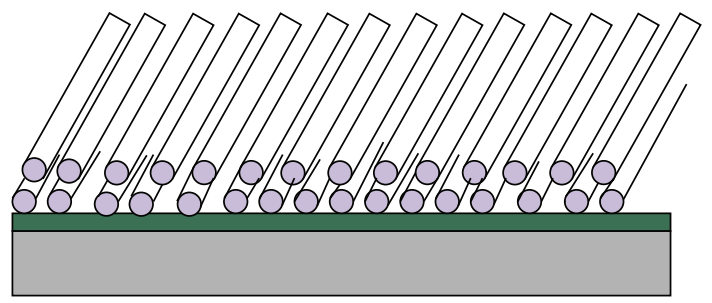

(b)

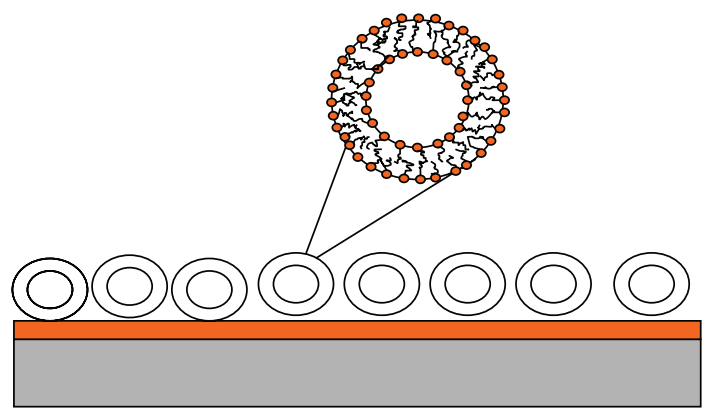

(c)

FIGURE 6: Sketch of supported membranes on the surface of an oscillating piezoelectric plate. (a) planar supported lipid bilayer : surface adjacent layer is anchored to the support;(b) LangmuirBlodgett film made of rodlike liquid crystal molecules; (c) supported membrane made of lipid vesicles.

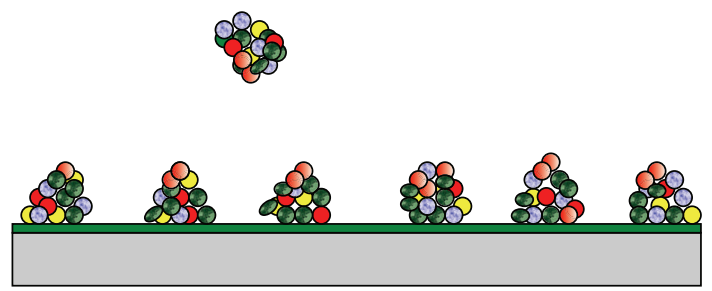

Figure 7: Protein layer adsorbed from a liquid phase onto a biochemically functionalized support.

biosensors, electronic nose and electronic tongue in which they can serve as sensitive elements.

This is also valid within the segregated (sublayer) structure model of adsorbed protein layers (Figure 7). The structure of the protein layer resembles a surfactant film composed of segregated head-and-tail regions. In this case, the relation between the upper and lower layer viscosities may vary in strong dependence of the phase state of protein and amphiphilic monolayer, respectively. 


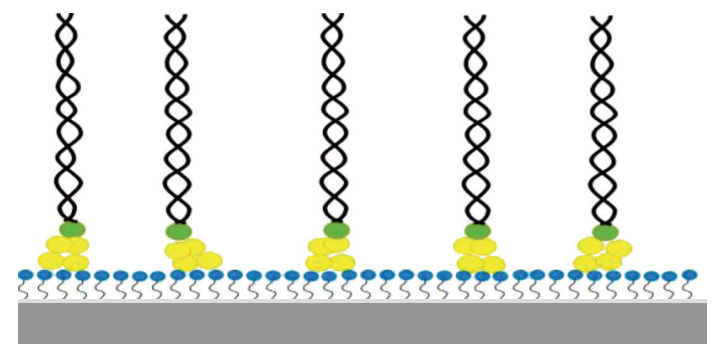

FIGURE 8: Multilayer architecture of biomolecular overlayer on the functionalized resonator surface.This QCM-based biosensor can be used, for example, for the detection of the DNA duplex formation and DNA-drug interactions. Binding of drugs increased energy dissipation (see for review [59]).

The dynamic (shear) viscosity of amphiphilic films is a key-importance rheological characteristic of a protein film or a fluid membrane, which is strongly dependent on temperature and phase state of the film, and on environmental conditions. Usually LB or SAM film acoustic sensors operate in vacuum or in a gaseous environment. In contrast, a lipid-water system or a protein layer adsorbed onto a solid surface in a fluid experiences "wet" conditions or a bulk aqueous medium at room temperature, which can change the viscosity of the film. In addition, the biomolecular layer adsorption process is extremely sensitive to the nature of the solid substrate in the sense that it can modify the overlayer structure schematically depicted in Figures 6(a) and 6(c). This is also valid within the segregated (sublayer) structure model of adsorbed protein layers (Figure 7). In this case, the relation between the upper and lower layer viscosities may vary in strong dependence of the phase state of protein and amphiphilic monolayer, respectively.

Amphiphilic bilayer films on supports can imitate the behavior of a bilayer lipid membrane. The constituent bilayer molecules are composed of hydrophilic groups attached to hydrophobic chains of different length. The phase diagrams of such amphiphilic molecules demonstrate a rich variety of properties and behavior depending on temperature and water content. Above the liquid crystalline-to-gel transition the hydrocarbon chains are approximately liquid and a bilayer membrane behaves like a two-dimensional fluid in the lateral plane due to the vanishing shear modulus of elasticity. Such a peculiar fluid is isotropic in its plane but it is anisotropic in the normal direction due to sublayered head-and-tail structure [19]. Viscous properties of the layer can be characterized by different viscosity values, namely, the surface viscosity, and a "bulk" shear viscosity [70].

The surface viscosity of a fluid amphiphilic film is often considered to be a two-dimensional analogue of bulk viscosity and defined as the coefficient of proportionality between the tangential force per unit length and the gradient in the flow velocity of the liquid. These averaged surface characteristics can be measured, for example, by an interface shear rheometer at air-liquid interfaces or by using the oscillating barrier method in a LB trough. In contrast to $\eta$, the "microscopic" shear viscosity may be defined as a contribution of fluid disordered chains. The "microviscosity" of the membrane core can be determined experimentally, for example, by a probe technique, and describe the local viscosity near the probe [71]. This "microviscosity" is often identified with the true membrane viscosity. However, this experimental technique could give rather different results with a strong dependence on the probe material and on the interaction between the probe and its local surroundings. Both of these viscosities may be changed after the deposition of an amphiphilic layer on the solid surface.

Both types of resonators (SAW and BAW) $[19,70]$ can be applied for the detection of phase transitions in biomolecular films adsorbed onto the surface of the sensor. These acoustic devices allow us to measure the acoustic response of the biofilm or supported lipid membrane in the lipid phase transition region where the viscosity of the membrane is subject to dramatic changes. For instance, during the liquid crystalline to gel transformation of a lipid membrane, the viscosity of the bilayer changes by more than 10 times, while the membrane density remains practically constant [70]. It is an interesting experimental fact that the two halves of a bilayer lipid membrane are so weakly coupled, that they can undergo a thermotropic phase transition independently. This must lead to changes in the SAW damping coefficient, while the corresponding SAW phase velocity shift due to the presence of the bilayer will be constant.

When the system is immersed in a water solution, anisotropy can arise from water trapping into the layer. This can result in a distinct overlayer viscosity in comparison with an air environment. This is especially important for protein adsorbed layers with nonuniform interfacial region structure and water distribution in the normal direction. These effects are also important for the lipid vesicles adsorption measured with QCM [72, 73].

In addition to its importance for biosensor applications, the study of the hydrodynamic modes of fluid amphiphilic films and adsorbed proteins are generally important for understanding the dynamics of lipid-water systems and the rheology of biological membranes. It is known, for instance, that living cell membranes, for example, in red blood cells experience shear stress in hydrodynamic flow through capillaries. As the lipid bilayer matrix of the membrane is a two-dimensional incompressible liquid adjacent to an elastic protein (spectrin-actin) network, the hydrodynamics of this layered structure are governed by the coupling of the fluid membrane to shear flows in an external bulk liquid. Recent efforts in measurements of hydrodynamic effects in QCM applications are reported in [74].

6.2. QCM in Electronic Nose Applications. Among artificial sensor systems, the array of electronic chemical sensors (electronic nose) working in gaseous environments and imitating the mammalian sense of smell remain, the primary instrumentation, although sensors operating in aqueous phase and having the same properties of electronic nose (the so-called electronic tongue) have been reported [74-82]. In many cases, the electronic tongue can be regarded as a wet 


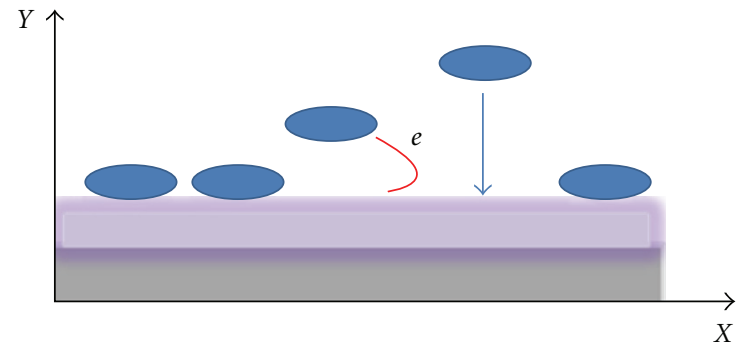

FIgURE 9: A sketch of the electrochemical detection of the redox enzyme reaction with charge transfer to the EQCM, a quartz crystal covered with a layer of electroconducting polymers on its surface and operating in a water solution with ions or buffer.

chemical counterpart of the electronic nose. Recently, the integration of the electronic nose, and the electronic tongue into a multisensor device has been proposed to improve the quality analysis of liquid samples and related volatile components. The simultaneous usage of these two types of sensors covered with the same sensing molecular compounds (metalloporphyrins) has been used by, for example, authors [75] to optimize the characteristics of the sensor.

A special type of quartz resonators, the so-called electrochemical QCM (EQCM), is a sensor enabling detection of chemical parameters via electrical conductivity measurements both in gaseous phase, vapours, and in various liquids. Typically the EQCM is based on usage of electroconducting polymer arrays as a smart coating for the sensor surface.

If the QCM device is used as a mass sensor in electronic tongue applications, the density and viscosity of the solution affect the resonance frequency of the oscillator. The classical result of Gordon and Kanazawa [51] is applicable here with a correction for a possible missing mass effect contribution [53]. The latter can be useful for interpreting QCM data in electronic tongue and biosensor applications since the correct estimation of the mass derived from the QCM response can be a crucial step towards using the microbalance as a sensor in complex biological liquids, where biomolecules or cells adsorbed from the solution make a soft layer on the receptive surface of the oscillator. In some situations, it is useful to combine the voltammetric electronic tongue (or EQCM) and QCM technique to relate the mass changes and charge transport problem in analytic chemistry applications and in situ electrochemical measurements [83]. For example, it has been reported elsewhere [76] that if a coating composed of redox active polymers, the QCM can monitor the mass changes which accompany the redox processes in polymer matrix. There the relationship between the charge, a measure of the total number of electrons produced or consumed in redox reactions, and mass transport has been derived from the combined QCM and voltammetry data.

Electronic noses based on the QCM device have been widely used as gas sensors to "smell" volatile organic compounds (VOCs) in food, olive oil, and wine industry applications as well as in health care and environmental monitoring, for security and safety purposes [77-83].
6.3. The Problem of Sliding Friction for Viscoelastic Adlayers. Successful applications of quartz resonators as a sensor element in electronic noses and electronic tongues (or EQCMs) bring up the fundamental question of how internal friction (effects of viscosity) and interfacial friction (slippage) can affect the QCM resonance frequency shift $\Delta f$ and, hence, the mass sensitivity. In cases when the overlayer material exhibits a viscoelastic behaviour, both internal and interfacial friction may contribute to energy dissipation. Depending on the situation, the former or the latter type of friction may dominate. Recently, a dynamic "slip/no-slip" test that can be applied to QCM thin films spectroscopy has been suggested elsewhere [62]. The test is based on the theoretical finding that the peak in the viscous dissipation $\Delta D$ depends on the frequency while the peak value of the $\Delta D$ dissipation factor caused by sliding friction is frequency independent. It has been shown [62] that the combined action of these two mechanisms may cause a nonmonotonic variation of the shift in the dissipation factor $\Delta D$ of quartz oscillator when adlayer parameters are varied.

The effects of interfacial slippage must be taken into account when the condition of rigid attachment of the adlayer to the oscillator surface is violated. A finite amount of slippage can arise either from incommensurate densities [84] or weak coupling between the aldayer and the substrate and influence both the resonance frequency $f$ and the oscillator dissipation $\Delta D$ (a comprehensive and extensive analysis of sliding friction can be found in the recent review by Krim [85] and in the book by Persson [84]).

For a quantitive characterization of slippage effects it is convenient to use the ratio of the inverse quality factor $\Delta(1 / Q) \equiv \Delta D$ to the shift $\Delta f$ of the QCM resonance frequency. This value has been introduced for the first time in the QCM community by Krim and Widom [86] as a "slip time":

$$
\tau=\frac{\Delta\left(Q^{-1}\right)}{4 \pi|\Delta f|} .
$$

According to the results of Krim and Widom [86], a partial decoupling of the overlayer should occur when $2 \pi f \tau \geq 0.5$. Such a relatively low decoupling threshold can lead to noticeable interfacial friction effects even in the case of molecularly thin films. In nanotribological QCMexperiments of Krim et al. [86, 87], the interfacial friction coefficient $\gamma_{f}$

$$
\gamma_{f}=\frac{1}{\tau} \sim m_{f}
$$

has been found be proportional to the surface mass density $m_{f}=\rho_{f} h_{f}$ of the film. The authors [86] attributed the "slip time" $\tau$ as a relaxation time associated with film-momentum fluctuations. This result can also be viewed as the definition of the slip time as the characteristic time it takes for the film velocity to decrease by a factor of $1 / e$. However, it has been shown [62] that for soft (viscoelastic) materials this result should be corrected because of additional viscous dissipation in the overlayers. In analogy with (21), in order to characterize the temporal QCM response when no-slip 
boundary conditions apply, another characteristic value can be introduced:

$$
\tau_{0}=-\frac{\Delta\left(Q^{-1}\right)}{4 \pi \Delta \tilde{f}}
$$

Here $\Delta \tilde{f}$ is negative and tilde denotes no-slip conditions. Defined in this way, the characteristic time $\tau_{0}$ can be attributed solely to the effects of internal friction (shear viscosity) on the damping of the QCM. One can find the coefficient of slippage, $\lambda \equiv 1 / \gamma$, from the experimentally measured $\tau_{S}$ value as follows:

$$
\lambda \approx \frac{\omega \tau_{0}-\omega \tau_{S}}{2 \pi \Delta \tilde{f} M} .
$$

Simultaneous measurements of $\Delta f$ and $\Delta D$ in QCM-D experiments at different frequencies open a way to estimate the coefficient of slippage, for example, by comparing the frequency behaviour of the dissipation factor at maximum [62] for various environmental parameters.

6.4. Future Outlook. Recent trends and the future outlook in the design, fabrication, and utilization of AW-based sensors span many different fields and interdisciplinary research. Sensor development stretches from nanoscale technology needs to terrestrial ecosystems (including the ecology of oceans and environment studies) and even extraterrestrial research. Surface acoustic wave- (SAW-) based devices are the most popular high frequency systems due to their extraordinary success in mobile and wireless communication, in electronics and the sensors industry $[88,89]$. These SAW piezoelectric oscillators [90] as well as BAW devices such as quartz crystal microbalance have now got a varied number of applications ranging from high precision gravimetrical measurements to biosensors. The selected examples are the usage of acoustic wave devices in the marine research $[5,6]$, coastal developments and biofoul control $[7,8]$, acousticcounterflow microfluidics by surface acoustic waves [2729], Life sciences and health care service [ $81,83,91,92]$, safety, security, and Man-Portable Threat Warning Systems [9], further development of a microbalance system for dust and water vapour detection in the Mars atmosphere [1-3] and AW-sensors for astrobiology research [4].

There is a rapid progress in the material science of new polymeric coatings and dynamic macromolecular assemblies for biosensor purposes (see, e.g., the excellent reviews of Lehn [93] and Sanchez and coworkers [94]). The frontier chemical strategy for the innovative structures manufacturing is the combination of integrated synthesis and selfassembly or nanobuilding block techniques [71, 93-96], usage of hybrid Metal Organic Frameworks (MOFs) [94], and nanoclay-based compounds [97], new porous materials [95], and bioinspired structures functional coatings [98$100]$.

The combination of novel polymeric materials, synthetic or natural, with the advanced surface science nanotechnologies (a polymer pen lithography for the surface patterning is a selected current example [101]) will be a further step towards the design of "smart" biosensors of new generation.
Valuable information on recent trends in sensors technology the reader can find also in the International Frequency Sensor Association web portal [10].

\section{Appendix}

\section{The List of (Selected) Companies Manufacturing AW Sensors}

(1) Q-sense (http://www.q-sense.com/diverse_use_of qcm_d_demonstrated_in_latest_research-209.asp).

(2) CENS (http://research.cens.ucla.edu/) UCLA's Center for Embedded Networked Sensing (CENS) with applications in the Terrestrial ecology.

(3) Nanofilm (http://www.nanofilm.de/) (SAW biosensors).

(4) E-QCM (http://ichf.edu.pl/offers/instrum/quartz .htm).

(5) QCM (http://www.elbatech.com/).

(6) QCM, EQCM of smaller size plus connection via Qpod to PC via USB port to computerize data; also Combination of QCM sensor and INFICON Sigma Instruments products (http://www.sig-inst.com/).

(7) EQCM company (http://www.meeck.pl/eqcm/en_ m106.html).

(8) DTI Defens Information Community (http://www .dtic.mil/dtic/).

(9) Honeywell (http://www51.honeywell.com/honeywell/) (Allied Signal Corporation, production of Metglas).

(10) Advalytix (http://www.Advalytix.de/) Microfluidic applications: SAW Technology can be used for pumping, dosing and mixing of reagents in low volumes.

(11) Microsensor Systems Inc. (MSI), the world leader in Surface Acoustic Wave (SAW) based chemical sensing (http://www.microsensorsystems.com/aboutus .html) and (http://www.microsensorsystems.com/).

(12) QCM100 and QCM200 (http://www.thinksrs.com/), Vacuum \& Analytical Instruments.

\section{Acknowledgments}

The author would like to express special gratitude to Professor Mats Jonson, colleague and the coauthor of all her QCM publications, for his help and critical reading of the manuscript. Financial support from the Swedish SSF is gratefully acknowledged.

\section{References}

[1] W. Gao, S. Osman, and J. Barengoltz, "Quantification of spore-forming bacteria carried by dust particles," in Proceedings of the 36th COSPAR Scientific Assembly, Jet Propulsion Laboratory, National Aeronautics and Space Administration, Beijing, China, July 2006. 
[2] E. Palomba, L. Colangeli, P. Palumbo, A. Rotundi, J. M. Perrin, and E. Bussoletti, "Performance of micro-balances for dust flux measurement," Advances in Space Research, vol. 29, no. 8, pp. 1155-1158, 2002.

[3] R. Battaglia, E. Palomba, P. Palumbo, L. Colangeli, and V. Della Corte, "Development of a micro-balance system for dust and water vapour detection in the Mars atmosphere," Advances in Space Research, vol. 33, no. 12, pp. 2258-2262, 2004.

[4] E. Robens and D. Möhlmann, "Planning of gravimetric investigations on Mars," Journal of Thermal Analysis and Calorimetry, vol. 76, no. 2, pp. 671-675, 2004.

[5] M. Berglin, J. Hedlund, C. Fant, and H. Elwing, "Use of surface-sensitive methods for the study of adsorption and cross-linking of marine bioadhesives," Journal of Adhesion, vol. 81, no. 7-8, pp. 805-822, 2005.

[6] http://research.cens.ucla.edu/about/annual_reports/CENSAnnualReport2008.pdf.

[7] S.-R. Hong, S.-J. Choi, H. D. Jeong, and S. Hong, "Development of QCM biosensor to detect a marine derived pathogenic bacteria Edwardsiella tarda using a novel immobilisation method," Biosensors and Bioelectronics, vol. 24, no. 6, pp. 1635-1640, 2009.

[8] P. J. Molino, O. M. Hodson, J. F. Quinn, and R. Wetherbee, "The quartz crystal microbalance: a new tool for the investigation of the bioadhesion of diatoms to surfaces of differing surface energies," Langmuir, vol. 24, no. 13, pp. 6731-6737, 2008.

[9] C. M. Harris, "Product review: seeing SAW potential," Analytical Chemistry, vol. 75, no. 15, pp. 355A-358A, 2003.

[10] "International Frequency Sensor Association (IFSA)," http://www.sensorsportal.com .

[11] L. A. Francis, J.-M. Friedt, C. Bartic, and A. Campitelli, "A SU-8 liquid cell for surface acoustic wave biosensors," vol. 5455 of Proceedings of SPIE, pp. 353-363, 2004.

[12] K. Länge, G. Blaess, A. Voigt, R. Götzen, and M. Rapp, "Integration of a surface acoustic wave biosensor in a microfluidic polymer chip," Biosensors and Bioelectronics, vol. 22, no. 2, pp. 227-232, 2006.

[13] K. Länge, B. E. Rapp, and M. Rapp, "Surface acoustic wave biosensors: a review," Analytical and Bioanalytical Chemistry, vol. 391, no. 5, pp. 1509-1519, 2008.

[14] H. Uberall, in Physical Acoustics: Principles and Methods, W. P. Mason and R. N. Thurston, Eds., Academic Press, New York, NY, USA, 1988.

[15] E. Gizeli, N. J. Goddard, C. R. Lowe, and A. C. Stevenson, "A love plate biosensor utilising a polymer layer," Sensors and Actuators B, vol. 6, no. 1-3, pp. 131-137, 1992.

[16] G. Kovacs and A. Venema, "Theoretical comparison of sensitivities of acoustic shear wave modes for (bio)chemical sensing in liquids," Applied Physics Letters, vol. 61, no. 6, pp. 639-641, 1992.

[17] J. Du, G. L. Harding, J. A. Ogilvy, P. R. Dencher, and M. Lake, "A study of Love-wave acoustic sensors," Sensors and Actuators A, vol. 56, no. 3, pp. 211-219, 1996.

[18] G. L. Harding, J. Du, P. R. Dencher, D. Barnett, and E. Howe, "Love wave acoustic immunosensor operating in liquid," Sensors and Actuators A, vol. 61, no. 1-3, pp. 279-286, 1997.

[19] M. V. Voinova, M. Jonson, and B. Kasemo, "Dynamics of viscous amphiphilic films supported by elastic solid substrates," Journal of Physics: Condensed Matter, vol. 9, no. 37, pp. 7799-7808, 1997.
[20] M. V. Voinova, A. M. Kosevich, and E. S. Syrkin, "Influence of Langmuir-Blodgett films on the characteristics of surfacewaves in a crystal," Acoustical Physics, vol. 39, pp. 500-501, 1993.

[21] H. Wohltjen and R. Dessy, "Surface acoustic wave probe for chemical analysis-I: introduction and instrument description," Analytical Chemistry, vol. 51, no. 9, pp. 1458-1464, 1979.

[22] C. Zimmermann, D. Rebière, C. Déjous, J. Pistré, E. Chastaing, and R. Planade, "A love-wave gas sensor coated with functionalized polysiloxane for sensing organophosphorus compounds," Sensors and Actuatorss B, vol. 76, no. 1-3, pp. 86-94, 2001.

[23] I. D. Avramov, A. Voigt, and M. Rapp, "Rayleigh SAW resonators using gold electrode structure for gas sensor applications in chemically reactive environments," Electronics Letters, vol. 41, no. 7, pp. 450-452, 2005.

[24] I. D. Avramov, K. Länge, S. Rupp, B. Rapp, and M. Rapp, "Polymer coating behavior of rayleigh-SAW resonators with gold electrode structure for gas sensor applications," IEEE Transactions on Ultrasonics, Ferroelectrics, and Frequency Control, vol. 54, no. 1, pp. 157-166, 2007.

[25] D. W. Branch and S. M. Brozik, "Low-level detection of a Bacillus anthracis simulant using Love-wave biosensors on $36^{\circ} \mathrm{YX} \mathrm{LiTaO}_{3}$," Biosensors and Bioelectronics, vol. 19, no. 8, pp. 849-859, 2004.

[26] M. Cecchini, S. Girardo, D. Pisignano, R. Cingolani, and F. Beltram, "Acoustic-counterflow microfluidics by surface acoustic waves," Applied Physics Letters, vol. 92, no. 10, pp. 104103-1-104103-3, 2008.

[27] J. W. Grate, S. J. Martin, and R. M. White, "Acoustic wavemicrosensors-part I," Analytical Chemistry, vol. 65, no. 21, pp. 940A-948A, 1993.

[28] K. Sritharan, C. J. Strobl, M. F. Schneider, A. Wixforth, and Z. Guttenberg, "Acoustic mixing at low Reynold's numbers," Applied Physics Letters, vol. 88, no. 5, pp. 1-3, 2006.

[29] A. Wixforth, "Acoustically driven programmable microfluidics for biological and chemical applications," Journal of the Association for Laboratory Automation, vol. 11, no. 6, pp. 399405, 2006.

[30] C. A. Grimes, K. G. Ong, K. Loiselle, et al., "Magnetoelastic sensors for remote query environmental monitoring," Smart Materials and Structures, vol. 8, no. 5, pp. 639-646, 1999.

[31] S. Schmidt and C. A. Grimes, "Elastic modulus measurement of thin films coated onto magnetoelastic ribbons," IEEE Transactions on Magnetics, vol. 37, no. 4, part 1, pp. 27312733, 2001.

[32] M. K. Jain and C. A. Grimes, "Effect of surface roughness on liquid property measurements using mechanically oscillating sensors," Sensors and Actuators A, vol. 100, no. 1, pp. 63-69, 2002.

[33] M. Zourob, K. G. Ong, K. Zeng, F. Mouffouk, and C. A. Grimes, "A wireless magnetoelastic biosensor for the direct detection of organophosphorus pesticides," Analyst, vol. 132, no. 4, pp. 338-343, 2007.

[34] C. Ruan, K. Zeng, O. K. Varghese, and C. A. Grimes, "Magnetoelastic immunosensors: amplified mass immunosorbent assay for detection of Escherichia coli O157:H7," Analytical Chemistry, vol. 75, no. 23, pp. 6494-6498, 2003.

[35] D. Kouzoudis and C. A. Grimes, "Remote query fluidflow velocity measurement using magnetoelastic thick-film sensors," Journal of Applied Physics, vol. 87, no. 9, pp. 63016303, 2000. 
[36] P. G. Stoyanov and C. A. Grimes, "Remote query magnetostrictive viscosity sensor," Sensors and Actuators A, vol. 80, no. 1, pp. 8-14, 2000.

[37] S. Wu, X. Gao, Q. Cai, and C. A. Grimes, "A wireless magnetoelastic biosensor for convenient and sensitive detection of acid phosphatase," Sensors and Actuators B, vol. 123, no. 2, pp. 856-859, 2007.

[38] L. G. Puckett, J. K. Lewis, A. Urbas, X. Cui, D. Gao, and L. G. Bachas, "Magnetoelastic transducers for monitoring coagulation, clot inhibition, and fibrinolysis," Biosensors and Bioelectronics, vol. 20, no. 9, pp. 1737-1743, 2005.

[39] J. Wan, H. Shu, S. Huang, et al., "Phase-based magnetoelastic wireless biosensors for detecting Bacillus anthracis spores," IEEE Sensors Journal, vol. 7, no. 3, pp. 470-477, 2007.

[40] C. Ruan, K. Zeng, O. K. Varghese, and C. A. Grimes, "A magnetoelastic bioaffinity-based sensor for avidin," Biosensors and Bioelectronics, vol. 19, no. 12, pp. 1695-1701, 2004.

[41] Q. Y. Cai and C. A. Grimes, "A remote query magnetoelastic pH sensor," Sensors and Actuators B, vol. 71, no. 1-2, pp. 112 $117,2000$.

[42] R. Guntupalli, J. Hu, R. S. Lakshmanan, T. S. Huang, J. M. Barbaree, and B. A. Chin, "A magnetoelastic resonance biosensor immobilized with polyclonal antibody for the detection of Salmonella typhimurium," Biosensors and Bioelectronics, vol. 22, no. 7, pp. 1474-1479, 2007.

[43] R. S. Lakshmanan, R. Guntupalli, S. Huang, et al., "Magnetoelastic material as a biosensor for the detection of salmonella typhimurium," in Proceedings of the Symposium V: Materials, Devices, and Characterization for Smart Systems, MRS Fall Meeting, Boston, Mass, USA, December 2008.

[44] L. G. Puckett, G. Barrett, D. Kouzoudis, C. Grimes, and L. G. Bachas, "Monitoring blood coagulation with magnetoelastic sensors," Biosensors and Bioelectronics, vol. 18, no. 5-6, pp. 675-681, 2003.

[45] M. V. Voinova and M. Jonson, "On theory of magnetoelastic sensors," unpublished.

[46] G. Sauerbrey, "Use of quartz vibrator for weighing thin layers and as a micro-balance," Zeitschrift fur Physik, vol. 155, no. 2, pp. 206-222, 1959.

[47] C. D. Stockbridge, "Effects of gas pressure on quartz-crystal microbalances," in Vacuum Microbalance Techniques, vol. 5, pp. 147-178, 1966.

[48] M. Rodahl, F. Höök, C. Fredriksson, et al., "Simultaneous frequency and dissipation factor QCM measurements of biomolecular adsorption and cell adhesion," Faraday Discussions, vol. 107, pp. 229-246, 1997.

[49] D. Johannsmann, "Studies of viscoelasticity with the QCM," in Piezoelectric Sensors, C. Steinem and A. Janshoff, Eds., pp. 49-110, Springer, New York, NY, USA, 1st edition, 2006.

[50] L. Daikhin, E. Gileadi, G. Katz, V. Tsionsky, M. Urbakh, and D. Zagidulin, "Influence of roughness on the admittance of the quartz crystal microbalance immersed in liquids," Analytical Chemistry, vol. 74, no. 3, pp. 554-561, 2002.

[51] K. K. Kanazawa and J. G. Gordon II, "Frequency of a quartz microbalance in contact with liquid," Analytical Chemistry, vol. 57, no. 8, pp. 1770-1771, 1985.

[52] B. Zimmermann, R. Lucklum, P. Hauptmann, J. Rabe, and S. Büttgenbach, "Electrical characterisation of high-frequency thickness-shear-mode resonators by impedance analysis," Sensors and Actuators B, vol. 76, no. 1-3, pp. 47-57, 2001.

[53] M. V. Voinova, M. Jonson, and B. Kasemo, "'Missing mass' effect in biosensor's QCM applications," Biosensors and Bioelectronics, vol. 17, no. 10, pp. 835-841, 2002.
[54] M. V. Voinova, M. Rodahl, M. Jonson, and B. Kasemo, "Viscoelastic acoustic response of layered polymer films at fluid-solid interfaces: continuum mechanics approach," Physica Scripta, vol. 59, no. 5, pp. 391-396, 1999.

[55] J. Limson, O. O. Odunuga, H. Green, F. Höök, and G. L. Blatch, "The use of a quartz crystal microbalance with dissipation for the measurement of protein-protein interactions: a qualitative and quantitative analysis of the interactions between molecular chaperones," South African Journal of Science, vol. 100, no. 11-12, pp. 678-682, 2004.

[56] D. Johannsmann, I. Reviakina, E. Rojas, and M. Gallego, Analytical Chemistry. In press.

[57] M. Rodahl, F. Höök, A. Krozer, P. Brzezinski, and B. Kasemo, "Quartz crystal microbalance setup for frequency and Qfactor measurements in gaseous and liquid environments," Review of Scientific Instruments, vol. 66, no. 7, pp. 3924-3930, 1995.

[58] M. Rodahl M, F. Höök, A. Kroze, and B. Kasemo, "Piezo-electric crystal microbalance Device," US patent no. US6006589, 1995.

[59] F. Höök and B. Kasemo, "The QCM technique for biomacromoelcular recognition: technical and theoretical aspects," $\mathrm{O}$. S. Wolfbeis, Ed., The Springer Series on Chemical Sensors and Biosensors, 2006.

[60] V. M. Mecea, "Is quartz crystal microbalance really a mass sensor?" Sensors and Actuators A, vol. 128, no. 2, pp. 270-277, 2006.

[61] M. Gavutis, S. Lata, and J. Piehler, "Probing 2-dimensional protein-protein interactions on model membranes," Nature Protocols, vol. 1, no. 4, pp. 2091-2103, 2006.

[62] M. V. Voinova, M. Jonson, and B. Kasemo, "On dissipation of quartz crystal microbalance as a mechanical spectroscopy tool," Spectroscopy, vol. 18, no. 4, pp. 537-544, 2004.

[63] M. Penza, P. Aversa, G. Cassano, et al., "Thin-film bulk-acoustic-resonator gas sensor functionalized with a nanocomposite Langmuir-Blodgett layer of carbon nanotubes," IEEE Transactions on Electron Devices, vol. 55, no. 5, pp. 1237-1243, 2008.

[64] M. Penza, M. A. Tagliente, P. Aversa, M. Re, and G. Cassano, "The effect of purification of single-walled carbon nanotube bundles on the alcohol sensitivity of nanocomposite Langmuir-Blodgett films for SAW sensing applications," Nanotechnology, vol. 18, no. 18, pp. 185502-185512, 2007.

[65] M. Benetti, D. Cannatà, F. Di Pietrantonio, V. Foglietti, and E. Verona, "Microbalance chemical sensor based on thin-film bulk acoustic wave resonators," Applied Physics Letters, vol. 87, no. 17, Article ID 173504, 3 pages, 2005.

[66] J. Bjurström, G. Wingqvist, V. Yantchev, and I. Katardjiev, "Temperature compensation of liquid FBAR sensors," Journal of Micromechanics and Microengineering, vol. 17, no. 3, pp. 651-658, 2007.

[67] G. Wingqvist, J. Bjurström, L. Liljeholm, V. Yantchev, and I. Katardjiev, "Shear mode AlN thin film electro-acoustic resonant sensor operation in viscous media," Sensors and Actuators B, vol. 123, no. 1, pp. 466-473, 2007.

[68] G. Wingqvist, J. Bjurström, A.-C. Hellgren, and I. Katardjiev, "Immunosensor utilizing a shear mode thin film bulk acoustic sensor," Sensors and Actuators B, vol. 127, no. 1, pp. 248-252, 2007.

[69] K. M. Lakin and J. S. Wang, "Acoustic bulk wave composite resonators," Applied Physics Letters, vol. 38, no. 3, pp. 125127, 1981. 
[70] M. V. Voinova and E. S. Syrkin, "Method for determining viscosity of liquid-crystalline Langmuir-Blodgett films," Crystallography Reports, vol. 43, no. 5, pp. 863-865, 1998.

[71] A. M. Dopico, Ed., Methods in Membrane Lipids, Humana Press, 2007.

[72] C. A. Keller and B. Kasemo, "Surface specific kinetics of lipid vesicle adsorption measured with a quartz crystal microbalance," Biophysical Journal, vol. 75, no. 3, pp. 13971402, 1998.

[73] C. A. Keller, K. Glasmästar, V. P. Zhdanov, and B. Kasemo, "Formation of supported membranes from vesicles," Physical Review Letters, vol. 84, no. 23, pp. 5443-5446, 2000.

[74] E. Tellechea, D. Johannsmann, N. F. Steinmetz, R. P. Richter, and I. Reviakine, "Model-independent analysis of QCM data on colloidal particle adsorption," Langmuir, vol. 25, no. 9, pp. 5177-5184, 2009.

[75] J. W. Gardner and P. N. Bartlett, "Brief history of electronic noses," Sensors and Actuators B, vol. B18, no. 1-3, part 1, pp. 211-220, 1994.

[76] C. S. S. R. Kumar, "Nanosystem characterization tools in the life sciences," in Nanotechnologies for the Life Sciences, vol. 3, p. 413, Wiley-VCH, New York, NY, USA, 2006.

[77] J. W. Gardner and P. N. Bartlett, Electronic Noses: Principles and Applications, Oxford University Press, Oxford, UK, 1999.

[78] E. R. Hirst, Y. J. Yuan, W. L. Xu, and J. E. Bronlund, "Bond-rupture immunosensors-a review," Biosensors and Bioelectronics, vol. 23, no. 12, pp. 1759-1768, 2008.

[79] S. Rösler, R. Lucklum, R. Borngräber, J. Hartmann, and P. Hauptmann, "Sensor system for the detection of organic pollutants in water by thickness shear mode resonators," Sensors and Actuators B, vol. 48, no. 1-3, pp. 415-424, 1998.

[80] T. Jacobs, G. Cama, M. Hartmann, et al., "Micro fluidic biosensor array for parallelized cell adhesion analysis during pathogenic infection," IEEE Sensors, pp. 1460-1463, 2008.

[81] A. Mantini, C. Di Natale, A. Macagnano, R. Paolesse, A. Finazzi-Agrò, and A. D'Amico, "Biomedical application of an electronic nose," Critical Reviews in Biomedical Engineering, vol. 28, no. 3-4, pp. 481-485, 2000.

[82] J. M. Beeley, C. Mills, P. A. Hammond, et al., "All-digital interface ASIC for a QCM-based electronic nose," Sensors and Actuators B, vol. 103, no. 1-2, pp. 31-36, 2004.

[83] C. Di Natale, R. Paolesse, A. Macagnano, et al., "Electronic nose and electronic tongue integration for improved classification of clinical and food samples," Sensors and Actuators B, vol. 64 , no. 1-3, pp. 15-21, 2000.

[84] B. N. J. Persson, Sliding Friction Sliding Friction: Physical Principles and Applications (NanoScience and Technology), Springer, New York, NY, USA, 2nd edition, 2000.

[85] J. Krim, "QCM tribology studies of thin adsorbed films," Nano Today, vol. 2, no. 5, pp. 38-43, 2007.

[86] J. Krim and A. Widom, "Damping of a crystal oscillator by an adsorbed monolayer and its relation to interfacial viscosity," Physical Review B, vol. 38, no. 17, pp. 12184-12189, 1988.

[87] T. Coffey and J. Krim, "Quartz-crystal microbalance studies of the slippage of solid and liquid krypton monolayers on metal(111) and $\mathrm{C}_{60}$ surfaces," Physical Review B, vol. 72, no. 23, Article ID 235414, 5 pages, 2005.

[88] C. K. Campbell, "Applications of surface acoustic and shallow bulk acoustic wave devices," Proceedings of the IEEE, vol. 77, no. 10, pp. 1453-1484, 1989.

[89] C. K. Campbell, Surface Acoustic Wave Devices for Mobile and Wireless Communications, Academic Press, Boston, Mass, USA, 1998.
[90] J. W. Grate and G. C. Frye, "Acoustic wave sensors," Sensors Update, vol. 2, no. 1, pp. 37-83, 2001.

[91] "Detection Method for Cancer Detection and Protein Investigation," Nanofilm Surface Analysis, http://nanofilm.de.

[92] J. R. Heath and M. E. Davis, "Nanotechnology and cancer," Annual Review of Medicine, vol. 59, pp. 251-265, 2008.

[93] J.-M. Lehn, "From supramolecular chemistry towards constitutional dynamic chemistry and adaptive chemistry," Chemical Society Reviews, vol. 36, no. 2, pp. 151-160, 2007.

[94] C. Sanchez, B. Julián, P. Belleville, and M. Popall, "Applications of hybrid organic-inorganic nanocomposites," Journal of Materials Chemistry, vol. 15, no. 35-36, pp. 3559-3592, 2005.

[95] C. Férey, C. Mellot-Draznieks, C. Serre, et al., "Chemistry: a chromium terephthalate-based solid with unusually large pore volumes and surface area," Science, vol. 309, no. 5743, pp. 2040-2042, 2005.

[96] M. Barboiu, A. Cazacu, M. Michau, R. Caraballo, C. ArnalHerault, and A. Pasc-Banu, "Functional organic-inorganic hybrid membranes," Chemical Engineering and Processing: Process Intensification, vol. 47, no. 7, pp. 1044-1052, 2008.

[97] C. Mousty, "Sensors and biosensors based on clay-modified electrodes-new trends," Applied Clay Science, vol. 27, no. 34, pp. 159-177, 2004.

[98] L. Zhai, F. C. Cebeci, R. E. Cohen, and M. F. Rubner, "Stable superhydrophobic coatings from polyelectrolyte multilayers," Nano Letters, vol. 4, no. 7, pp. 1349-1353, 2004.

[99] C. Sanchez, H. Arribart, and M. M. G. Guille, "Biomimetism and bioinspiration as tools for the design of innovative materials and systems," Nature Materials, vol. 4, no. 4, pp. 277-288, 2005.

[100] C. Tamerler and M. Sarikaya, Eds., "Molecular biomimetics," Materials Research Society, vol. 33, no. 5, 2008.

[101] F. Huo, Z. Zheng, G. Zheng, L. R. Giam, H. Zhang, and C. A. Mirkin, "Polymer pen lithography," Science, vol. 321, no. 5896, pp. 1658-1660, 2008. 

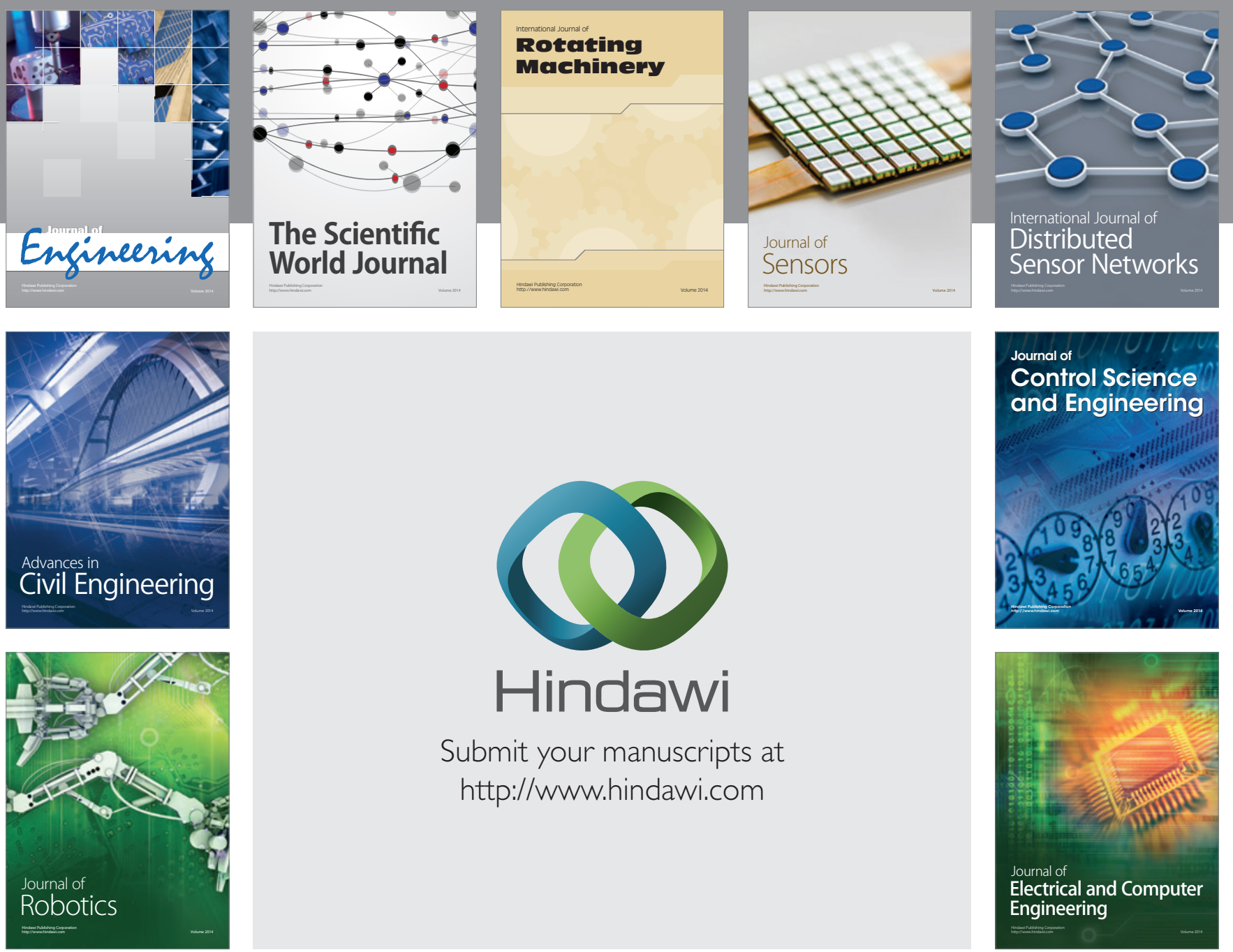

Submit your manuscripts at

http://www.hindawi.com
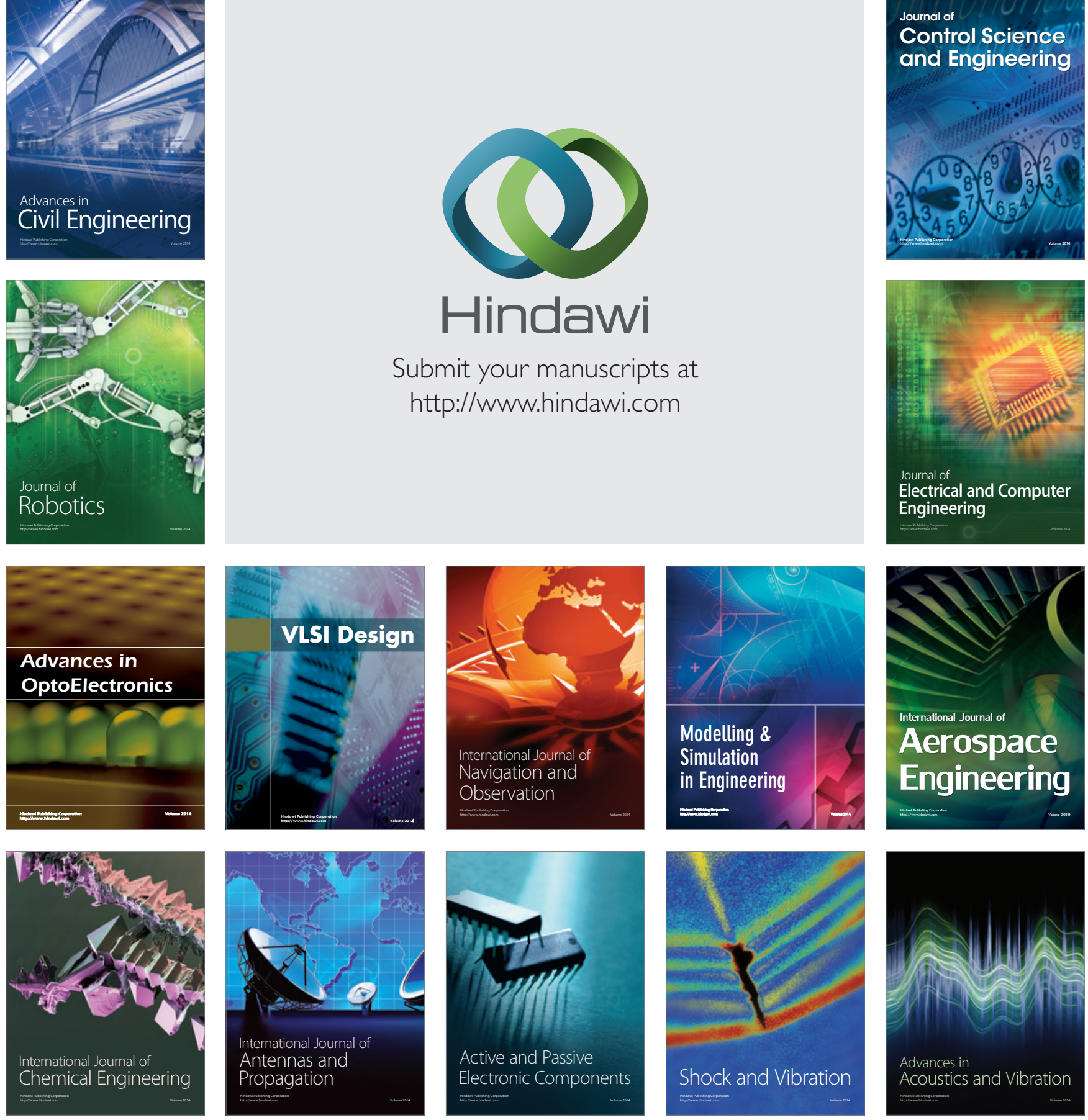